\title{
The basin of attraction method for a kind of nonlinear elliptic equations
}

\section{Yan-qing Feng*}

\author{
"Correspondence: fengyq@czu.cn \\ Department of Mathematics, \\ Changzhou Institute of Technology, \\ Changzhou, Jiangsu 213002, \\ P.R. China
}

\begin{abstract}
In this paper, a new sufficient condition of the existence and uniqueness of the second order elliptic boundary value problem is given by using the basin of attraction. Some known existent results on the existence of solutions of the nonlinear elliptic equation are generalized.
\end{abstract}

MSC: $35 \mathrm{C} 15$

Keywords: the basin of attraction; the second order elliptic equation; inequality; homeomorphism

\section{Introduction}

We will study the existence of solution for the second order elliptic equation

$$
\Delta u=f(x, u)
$$

where $\Delta$ denotes the $n$-dimensional Laplacian.

In 1976, Elcrat [1] derived an a priori estimate for the elliptic operator $L_{0} u$ defined by

$$
L_{0} u=a_{i j} u_{x_{i} x_{j}}+a_{i} u_{x_{i}}-a u
$$

and applied the result in the semilinear elliptic equation

$$
L_{0} u=a_{i j} u_{x_{i} x_{j}}+a_{i} u_{x_{i}}=f(x, u)
$$

to obtain the following existence theorem.

Theorem 1.1 [1] Suppose that $f$ satisfies the hypotheses of [1, Lemma 9] and that

(1) $\inf f_{u}^{\prime} \geq \sqrt{\lambda} S-\lambda v^{2}+\varepsilon, \varepsilon>0$

(2) $f_{u}^{\prime}=\mathrm{O}(u)$.

Then there is a unique solution of the equation $P u=L_{0} u-f(x, u)=0$ in $W_{2,0}^{2}(\Omega)$, where $v$ is a positive constant, $S=\sup \left|a_{i}-\left(a_{i j}\right)_{x_{j}}\right|, \lambda$ will be explained by the following paper.

Comparing (1.1) with (1.2), we have $a_{i j}=1, a_{i}=0$, then (1.2) becomes (1.1), and Theorem 1.1 can be expressed.

Corollary 1.1 [1] Suppose that $f$ satisfies the hypotheses of [1, Lemma 9] and that 
(1) $\inf f_{u}^{\prime} \geq-\lambda$;

(2) $f_{u}^{\prime}=\mathrm{O}(u)$.

Then there is a unique solution of the equation $P u=\Delta u-f(x, u)=0$ in $W_{2,0}^{2}(\Omega)$.

In this paper, we will give a new set of sufficient condition for the existence and uniqueness of the second order elliptic equation (1.1), to which Section 3 is devoted. In our main Theorem 3.1, we replace Elcrat's condition (2) by the condition

(2) for any $u \in W_{2,0}^{2}(\Omega), \int_{1}^{+\infty} \frac{d s}{1+N(s)+Q(s) N(s)}=\infty$, where

$N(s)=\sup _{\|u\|_{0} \leq s}\left\|\left[\Delta u-f_{u}^{\prime}(x, u)\right]^{-1}\right\|, Q(s)=\sup _{\Omega \times R} f_{u}^{\prime}(x, u)$ and Theorem 1.1 is a corollary of our main theorems.

Our proofs are different from the proofs given by Elcrat [1]. In Section 2 some functional analytic preliminaries are stated and some inequalities are derived in Section 3, which are useful for the proofs of our main theorems.

\section{Preliminaries}

We denote by $X, Y$ Banach spaces, $D$ an open connected subset of $X$. The following theorems will be employed to prove our main theorems.

Theorem 2.1 [2] Let $f: D \subset X \rightarrow Y$ be a $C^{1}$ mapping and a local homeomorphism, let $x_{0} \in D$. Then for any $x \in D$, the path-lifting problem

$$
\left\{\begin{array}{l}
f\left(\gamma_{x}(t)\right)=f\left(x_{0}\right)+e^{-t}\left(f(x)-f\left(x_{0}\right)\right), \quad t \in R \\
\gamma_{x}(0)=x, \quad \gamma_{x}(t) \in D
\end{array}\right.
$$

has a unique continuous solution $t \rightarrow \gamma_{x}(t)$ defined on the maximal open interval $I_{x}=$ $\left(t_{x^{-}}, t_{x^{+}}\right),-\infty \leq t_{x^{-}}, t_{x^{+}} \leq+\infty$. Moreover, the set $\left\{(x, t) \in D \times R: t \in I_{x}\right\}$ is open in $D \times R$ and the mapping $(x, t) \rightarrow \gamma_{x}(t)$ is continuous.

Definition 2.1 [2] In the setting of Theorem 2.1, the basin of attraction of $x_{0}$ is the set

$$
A=\left\{x \in D: t_{x^{+}}=+\infty, \lim _{t \rightarrow+\infty} \gamma_{x}(t)=x_{0}\right\}
$$

Theorem 2.2 [3] Let $f: X \rightarrow Y$ be a local homeomorphism. Then $f$ is a global homeomorphism of $X$ onto $Y$ if and only if $\gamma_{x}(t)$ is defined on $R$ for all $x \in A$, namely, $\gamma_{x}(t)$ can also be extended to $-\infty$.

\section{Main theorems}

Consider the second order elliptic boundary value problem

$$
\left\{\begin{array}{l}
\Delta u=f(x, u) \\
u(x)=0, \quad x \in \partial \Omega
\end{array}\right.
$$

where $f(x, u)$ is measurable in $x$ for all $u$ and has continuous partial derivatives in $u$ for almost all $x$.

We assume that $\Omega$ is a bounded domain in $R^{n}$ with piecewise smooth boundary $\partial G$ whose principal curvatures are bounded, and we assume that the function $u$ has square summable second derivatives on the domain $\Omega$. 
We will first study the action of the differential operator $L$

$$
L u=\Delta u-a(x) u
$$

on the class $W_{2,0}^{2}(\Omega)$, which may be defined as the closure of functions in $C_{2}(\bar{\Omega})$ that vanish on $\partial G$, where $a(x)$ is for the bounded measurable functions defined in $\Omega$.

With the above assumption $L$ may be thought of as a linear operator mapping $W_{2,0}^{2}(\Omega)$ into $L_{2}(\Omega)$. Let $W_{2,0}^{2}(\Omega)$ be the Hilbert space with the norm

$$
\left(\|u\|_{2}\right)^{2}=\int_{\Omega}\left(u^{2}+|\nabla u|^{2}+\left|D^{2} u\right|\right) d x
$$

where $\left|D^{2} u\right|^{2}$ denotes the sum of the squares of the second derivatives.

Our goal is then to establish an inequality of the form

$$
\|u\|_{2} \leq C\left(\|u\|_{0}+\|\Delta u\|_{0}\right)
$$

for $u$ in $W_{2,0}^{2}(\Omega)$. In order to obtain the desired results we will make use of the lowest eigenvalue $\lambda$ for the Laplacian with homogeneous boundary conditions on $\Omega$, which is given by

$$
\lambda=\inf \frac{\int_{\Omega}(\nabla u)^{2}}{\int_{\Omega} u^{2}},
$$

where the infimum is taken over $u$ in $W_{2}^{1}(\Omega)$.

Let

$$
m_{0}=\inf _{x \in \Omega} a(x) .
$$

Corollary 3.1 If $\inf _{x \in \Omega} a(x)>-\lambda$, then the inequality

$$
\|u\|_{2} \leq C\left(\|u\|_{0}+\|\Delta u\|_{0}\right)
$$

holds, where $C=\left(\frac{\varepsilon}{2}+\lambda+\frac{1}{\varepsilon}+1\right)^{1 / 2}$.

Proof From [4, Chapter 2, Section 8], we have

$$
\int_{\Omega}\left|D^{2} u\right|^{2} d x \leq \int_{\Omega}(\Delta u)^{2} d x,
$$

then for any $\varepsilon>0$, we can get

$$
\begin{aligned}
& \int_{\Omega}(\nabla u)^{2} d x+\int_{\Omega} a u^{2} d x \leq-\int_{\Omega} u L u d x \leq \frac{\varepsilon}{2} \int_{\Omega} u^{2} d x+\frac{1}{2 \varepsilon} \int_{\Omega}(L u)^{2} d x, \\
& \int_{\Omega}(\nabla u)^{2} d x+\int_{\Omega} a u^{2} d x \leq\left(\frac{\varepsilon}{2}+\frac{1}{\varepsilon}\right) \int_{\Omega} u^{2} d x+\frac{1}{\varepsilon} \int_{\Omega}(\Delta u)^{2} d x
\end{aligned}
$$


if $-\lambda<m_{0}<0$, and from (3.3), it follows that

$$
\begin{aligned}
& \int_{\Omega}(\nabla u)^{2} d x-\lambda \int_{\Omega} u^{2} d x \leq\left(\frac{\varepsilon}{2}+\frac{1}{\varepsilon}\right) \int_{\Omega} u^{2} d x+\frac{1}{\varepsilon} \int_{\Omega}(\Delta u)^{2} d x \\
& \int_{\Omega}(\nabla u)^{2} d x \leq\left(\frac{\varepsilon}{2}+\frac{1}{\varepsilon}+\lambda\right) \int_{\Omega} u^{2} d x+\frac{1}{\varepsilon} \int_{\Omega}(\Delta u)^{2} d x
\end{aligned}
$$

So

$$
\begin{aligned}
\left(\|u\|_{2}\right)^{2} & =\int_{\Omega}\left(u^{2}+|\nabla u|^{2}+\left|D^{2} u\right|\right) d x \\
& \leq\left(\frac{\varepsilon}{2}+1+\lambda+\frac{1}{\varepsilon}\right) \int_{\Omega} u^{2}+\left(\frac{1}{\varepsilon}+1\right) \int_{\Omega}(\Delta u)^{2}
\end{aligned}
$$

and if $m_{0} \geq 0$, then

$$
\int_{\Omega}(\nabla u)^{2} d x \leq\left(\frac{\varepsilon}{2}+\frac{1}{\varepsilon}\right) \int_{\Omega} u^{2} d x+\frac{1}{\varepsilon} \int_{\Omega}(\Delta u)^{2} d x
$$

So

$$
\begin{aligned}
\left(\|u\|_{2}\right)^{2} & =\int_{\Omega}\left(u^{2}+|\nabla u|^{2}+\left|D^{2} u\right|\right) d x \\
& \leq\left(\frac{\varepsilon}{2}+1+\frac{1}{\varepsilon}\right) \int_{\Omega} u^{2}+\left(\frac{1}{\varepsilon}+1\right) \int_{\Omega}(\Delta u)^{2} .
\end{aligned}
$$

Set $C=\left(\frac{\varepsilon}{2}+\lambda+\frac{1}{\varepsilon}+1\right)^{1 / 2}$, we have

$$
\|u\|_{2} \leq C\left(\|u\|_{0}+\|\Delta u\|_{0}\right)
$$

The corollary is proved.

We express (3.1) in the form

$$
P(u)=\Delta u-f(x, u)
$$

and (3.1) is equivalent to the operator equation

$$
P(u)=0, \quad u \in W_{2,0}^{2}(\Omega) .
$$

For any $u, \phi \in W_{2,0}^{2}(\Omega)$, we have

$$
P^{\prime}(u)(\phi)=\Delta \phi-f_{u}^{\prime}(x, u) \phi
$$

Now, we will show our main theorems.

\section{Theorem 3.1 Suppose}

(1) $\inf _{\Omega \times R} f_{u}^{\prime}>-\lambda$; 
(2)

$$
\text { for any } u \in W_{2,0}^{2}(\Omega), \quad \int_{1}^{+\infty} \frac{d s}{1+N(s)+Q(s) N(s)}=\infty \text { holds }
$$

where $Q(u)=\sup _{\Omega \times R} f_{u}^{\prime}(x, u), N(u)=\sup \left\|\left[\Delta u-f_{u}^{\prime}(x, u)\right]^{-1}\right\|$.

Then there is a unique solution of the equation

$$
P(u)=0
$$

in $W_{2,0}^{2}(\Omega)$.

Proof The condition $\inf _{\Omega \times R} f_{u}^{\prime}(x, u)>-\lambda$ implies that zero is not an eigenvalue of $\Delta \phi-$ $f_{u}^{\prime}(x, u) \phi=0$, so for every $u \in W_{2,0}^{2}(\Omega)$, the operator $P^{\prime}(u)=\Delta u-f_{u}^{\prime} I$ is invertible and $P$ is a local homeomorphism from $W_{2,0}^{2}(\Omega)$ onto $L_{2}(\Omega)$, where $I$ denotes the identical operator.

Denote $\left[\Delta+f_{u}^{\prime}(x, u) I\right]^{-1} \varphi=\psi$. From Corollary 3.1, we have

$$
\begin{aligned}
\|\psi\| & \leq C\left(\|\psi\|_{0}+\|\Delta \psi\|_{0}\right)=C\left(\left\|\left[\Delta-f_{u}^{\prime}(x, u) I\right]^{-1} \varphi\right\|+\left\|\Delta\left[\Delta-f_{u}^{\prime}(x, u) I\right]^{-1} \varphi\right\|\right) \\
& \leq C\left(N(\|u\|)\|\varphi\|+\|\varphi\|+\left\|f_{u}^{\prime}(x, u)\right\|\left\|\left[\Delta-f_{u}^{\prime}(x, u) I\right]^{-1} \varphi\right\|\right) \\
& \leq C\left(N(\|u\|)\|\varphi\|+\|\varphi\|+\sup \left\|f_{u}^{\prime}(x, u)\right\| N(\|u\|)\|\varphi\|\right) \\
& \leq C(N(\|u\|)\|\varphi\|+\|\varphi\|+Q(\|u\|) N(\|u\|)\|\varphi\|) .
\end{aligned}
$$

So we obtain

$$
\left\|P^{\prime}(u)^{-1}\right\|=\left\|\left[\Delta-f_{u}^{\prime}(x, u) I\right]^{-1}\right\| \leq C(1+N(\|u\|)+Q(\|u\|) N(\|u\|)) .
$$

Consider the path-lifting problem for the mapping $P$ :

$$
\left\{\begin{array}{l}
P\left(\gamma_{u}(t)\right)-P\left(u_{0}\right)=e^{-t}\left(P(u)-P\left(u_{0}\right)\right) \\
\gamma_{u}(0)=u
\end{array}\right.
$$

It is clear that

$$
\gamma_{u}^{\prime}(t)=-\left[P^{\prime}\left(\gamma_{u}(t)\right)\right]^{-1} e^{-t}\left(P(u)-P\left(u_{0}\right)\right) .
$$

Then

$$
\begin{aligned}
& \begin{aligned}
\left\|\gamma_{u}(t)-\gamma_{u}(0)\right\| & =\left\|\int_{0}^{t} \gamma_{u}^{\prime}(t) d t\right\| \leq \int_{0}^{t}\left\|\gamma_{u}^{\prime}(t)\right\| d t \\
& =\int_{0}^{t}\left\|\left[P^{\prime}\left(\gamma_{u}(t)\right)\right]^{-1} e^{-t}\left(P(u)-P\left(u_{0}\right)\right)\right\| d t \\
& \leq C \int_{0}^{t}\left|1+N\left(\gamma_{u}(t)\right)+Q\left(\gamma_{u}(t)\right) N\left(\gamma_{u}(t)\right)\right| e^{-t}\left\|P(u)-P\left(u_{0}\right)\right\| d t,
\end{aligned} \\
& \left\|\gamma_{u}(t)\right\| \leq\left\|\gamma_{u}(0)\right\|+C \int_{0}^{t}\left|1+N\left(\gamma_{u}(t)\right)+Q\left(\gamma_{u}(t)\right) N\left(\gamma_{u}(t)\right)\right| e^{-t}\left\|P(u)-P\left(u_{0}\right)\right\| d t .
\end{aligned}
$$


Using the Gronwall inequality, we have

$$
\int_{\left\|\gamma_{u}(0)\right\|}^{\left\|\gamma_{u}(t)\right\|} \frac{d s}{1+N(s)+Q(s) N(s)} \leq C \int_{0}^{t} e^{-t}\left\|P(u)-P\left(u_{0}\right)\right\| d t \leq C\left\|P(u)-P\left(u_{0}\right)\right\|
$$

which, together with (3.4), implies $\exists M>0$

$$
\left\|\gamma_{u}(t)\right\| \leq M, \quad t>0
$$

By Theorem 2.2, now we need only show that $\gamma_{u}(t)$ can be extended to $-\infty$.

Let $g(-t)=\gamma_{u}(t), t \in(b, 0], b<0$. Then for $t_{1}, t_{2} \in(b, 0]$

$$
\begin{aligned}
\left\|\gamma_{u}\left(t_{1}\right)-\gamma_{u}\left(t_{2}\right)\right\| & =\left\|g\left(-t_{1}\right)-g\left(-t_{2}\right)\right\|=\int_{-t_{2}}^{-t_{1}}\left\|g^{\prime}(s)\right\| d s \\
& =\int_{-t_{2}}^{-t_{1}}\left\|P^{\prime}\left(\gamma_{u}(t)\right)^{-1}\right\| e^{s}\left\|P(u)-P\left(u_{0}\right)\right\| d s \\
& \leq C \int_{-t_{2}}^{-t_{1}}|1+N(M)+Q(M) N(M)| e^{s}\left\|P(u)-P\left(u_{0}\right)\right\| d s \\
& \leq C(1+N(M)+Q(M) N(M)) e^{b}\left\|P(u)-P\left(u_{0}\right)\right\|\left|t_{1}-t_{2}\right| .
\end{aligned}
$$

So $\gamma_{u}(t)$ is Lipschitz continuous on $(-b, 0]$ and $\gamma_{u}(t)$ can be extended to $-\infty$, that is to say, $P$ is a diffeomorphism from $W_{2,0}^{2}(\Omega)$ to $L_{2}(\Omega)$ and the theorem is proved.

Corollary 3.2 Assume that $f$ satisfies:

(1) $\inf _{\Omega \times R} f_{u}^{\prime}>-\lambda$;

(2) Uniformly in $x,\left\|f_{u}^{\prime}\right\| \leq \omega(\|u\|)$, where $\omega$ is a continuous map satisfying

$$
\int_{a}^{\infty} \frac{d t}{\omega(t)}=\infty
$$

Then there is a unique solution of $(3.1)$ in $W_{2,0}^{2}(\Omega)$.

Proof We have from Theorem 1 in [1]

$$
\left\|\left[P^{\prime}(u)\right]^{-1}\right\| \leq \alpha \sup _{\Omega}\left|f_{u}^{\prime}(x, u(x))\right|+\beta
$$

for positive constants $\alpha, \beta$.

From (3.5),

$$
\int_{c}^{\infty} \frac{d t}{\alpha \sup \left|f_{u}^{\prime}\right|+\beta}=\infty
$$

By Theorem 3.1, there is a unique solution of $(3.1)$ in $W_{2,0}^{2}(\Omega)$.

Corollary 3.3 (Elcrat) Suppose that $P$ is given by $P u=\Delta u-f(x, u)$, that $f$ satisfies (1) $\inf _{\Omega \times R} f_{u}^{\prime}>-\lambda$, (2) $\left\|f_{u}^{\prime}\right\|=\mathrm{O}(\|u\|)$, uniformly in $x$.

Then there is a unique solution of the equation $P u=0$ in $W_{2,0}^{2}(\Omega)$. 
Proof Condition (2) implies that $\int_{a}^{\infty} \frac{d t}{\omega(t)}=\infty$ holds. The result of Elcrat in [5] becomes a special case of Theorem 3.1.

\section{Competing interests}

The author declares to have no competing interests.

\section{Author's contributions}

The author read and approved the final manuscript.

\section{Acknowledgements}

The author is grateful to the referees for their comments and references, which improved the paper. The work has been supported by the Natural Science Foundation of Jiangsu (13KJD110001).

Received: 15 March 2014 Accepted: 24 July 2014 Published online: 24 September 2014

\section{References}

1. Elcrat, AR: Constructive existence for semilinear elliptic equations with discontinuous coefficients. SIAM J. Math. Anal. 5, 663-672 (1974)

2. Gorni, G: A criterion of invertibility in the large for local diffeomorphisms between Banach spaces. Nonlinear Anal. 21, 43-47 (1993)

3. Marco, GD, Gorni, G, Zampieri, G: Global inversion of function: an introduction. Nonlinear Differ. Equ. Appl. 1, 229-248 (1994)

4. Ladyzhenskaya, OA, Uraltseva, NN: Linear and Quasilinear Elliptic Equations, pp. 134-145. Academic Press, New York (1968)

5. Feng, YQ, Wang, ZY, Wen, CJ: Global homeomorphism and applications to the existence and uniqueness of solutions of some differential equations. Adv. Differ. Equ. (2014). doi:10.1186/1687-1847-2014-52

doi:10.1186/s13661-014-0190-7

Cite this article as: Feng: The basin of attraction method for a kind of nonlinear elliptic equations. Boundary Value Problems 2014 2014:190.

\section{Submit your manuscript to a SpringerOpen ${ }^{\circ}$ journal and benefit from:}

- Convenient online submission

- Rigorous peer review

- Immediate publication on acceptance

- Open access: articles freely available online

- High visibility within the field

- Retaining the copyright to your article 